\title{
Gemella haemolysans
}

National Cancer Institute

\section{Source}

National Cancer Institute. Gemella haemolysans. NCI Thesaurus. Code C86891.

A species of facultatively anaerobic, Gram positive, cocci shaped bacteria assigned to the phylum Firmicutes. This species does not form endospores, is oxidase and catalase negative, and non motile. G. morbillorum may be found in the mucous membranes of the oral cavity and the upper respiratory tract. It is an opportunistic pathogen and a causative agent of endocarditis. 